for patients with PEF, the survival for those with REF increased over the study period, a trend that was not seen for PEF patients. As evidence accumulates that overall survival among patients with heart failure is increasing, the authors highlight the need for a therapeutic strategy to tackle the increasingly important problem of heart failure with PEF.

Original article Owan TE et al. (2006) Trends in prevalence and outcome of heart failure with preserved ejection fraction. N Engl J Med 355: 251-259

\section{Slow-pathway modification for AVNRT is sufficient to cure AF in select patients}

Atrioventricular nodal re-entrant tachycardia (AVNRT) can trigger atrial fibrillation (AF), but the incidence of AVNRT as a cause of AF is unclear. Identification of patients with AVNRT could improve the choice of catheter ablation strategy employed for AF. To determine the incidence of AVNRT-induced AF, Sauer et al. studied 629 consecutive patients referred for catheter ablation of AF from November 1998 to March 2005.

Inducible AVNRT was found in 24 patients at the initial procedure, and in a further 3 patients upon presentation for repeat ablation $(4.3 \%$ in total). None of the patients with AVNRT had a history of regular palpitations or regular narrow complex tachycardia. Patients with AVNRT tended to be younger than those without. In 13 patients, AVNRT was the only observed trigger for AF; these patients did not, therefore, undergo pulmonary vein isolation following slow-pathway modification. After a mean follow-up of 21.4 months, 21 (87.5\%) of the 24 patients initially identified with AVNRT had no recurrent $A F$, and no longer required antiarrhythmic medication. By contrast, only 329 (54.7\%) patients without AVNRT were cured of AF after a single procedure. Slow-pathway modification for AVNRT resolved the AF in the three patients in whom AVNRT was identified at second procedure.

The authors conclude that the success rate for curing AF is higher in patients with AVNRT than in those without, and that in a subset of patients resolving AVNRT is sufficient to cure AF. That three patients required a second visit before discovery of AVNRT emphasizes the need to ensure correct diagnosis, say the authors, especially as treatment for AVNRT cured AF in these patients.

Original article Sauer WH et al. (2006) Atrioventricular nodal reentrant tachycardia in patients referred for atrial fibrillation ablation. Circulation 114: 191-195

\section{Raloxifene reduces breast cancer, but not cardiovascular, risk in women}

In 1998, the Raloxifene Use for The Heart study was launched to investigate whether the selective estrogen-receptor modulator raloxifene reduces the risk of clinical coronary events and/or invasive breast cancer.

This randomized, double-blind trial recruited 10,001 postmenopausal women (mean age 67.5 years) with established coronary heart disease (CHD) or multiple CHD risk factors. Of these women, 5,044 were randomly allocated to receive $60 \mathrm{mg}$ raloxifene daily; the rest received placebo. The incidence of invasive breast cancer and coronary events (including death from coronary causes, hospitalization for an acute coronary syndrome, and nonfatal myocardial infarction) was recorded over a median follow-up of 5.56 years.

Raloxifene treatment approximately halved the risk of invasive breast cancer (absolute risk reduction 1.2 cases per 1,000 women treated for 1 year), and was associated with a $33 \%$ lower incidence of all breast cancer, compared with placebo. Markedly fewer clinical vertebral fractures occurred in patients given raloxifene than in controls. Raloxifene treatment did not affect the risk of primary coronary events, but treated patients were nearly 1.5 times more likely to suffer venous thromboembolism or fatal stroke than controls. Hot flashes, leg cramps, and peripheral edema were also associated with raloxifene treatment.

The authors conclude that, although raloxifene treatment reduces the risk of invasive breast cancer, it does not reduce risk of coronary events in postmenopausal women with, or at increased risk for, CHD. Patients and clinicians must consider both the benefits and risks of raloxifene when contemplating its use.

Original article Barrett-Connor E et al. (2006) Effects of raloxifene on cardiovascular events and breast cancer in postmenopausal women. N Engl J Med 355: 125-137 